\title{
Utilidad de la enteroscopia transoperatoria en hemorragia digestiva grave originada en el intestino delgado. Descripción de una técnica utilizando gastroduodenoscopio y presentación de tres casos clínicos
}

\author{
Usefulness of intraoperative enteroscopy in severe gastrointestinal \\ bleeding originating in the small intestine. Description of a technique \\ using gastroduodenoscope and presentation of three clinical cases
}

Mauro Soto Granados, ${ }^{*}$ Blanca Esther Maldonado Palacios**

\section{Palabras clave: \\ Enteroscopia \\ transoperatoria, \\ hemorragia digestiva, \\ intestino delgado.}

Key words:

Intraoperative

enteroscopy,

gastrointestinal

bleeding, small bowel.
* Jefe del Servicio de Cirugía.

** Jefa del Servicio de

Anestesia.

Hospital Privado Médica Farallón.

Recibido: 09/06/2016 Aceptado: 28/09/2016

\section{RESUMEN}

La hemorragia digestiva grave originada en el yeyuno e íleon es poco frecuente, pero constituye una seria amenaza para la vida. En estas circunstancias, la localización rápida y precisa del sitio de sangrado es fundamental para prevenir y/o disminuir la morbimortalidad por choque hipovolémico. La revisión endoscópica del intestino delgado, aun en condiciones electivas, no es sencilla y requiere de recursos diagnósticos sofisticados de difícil disposición en la mayoría de los hospitales de nuestro país, máxime tratándose de situaciones urgentes. Para estos casos, resulta de gran utilidad la enteroscopia transoperatoria practicada con un gastroduodenoscopio, un recurso diagnóstico disponible actualmente en muchas instituciones de salud públicas y privadas. En este reporte se describe una técnica de enteroscopia transoperatoria, se informan tres casos clínicos diagnosticados exitosamente a través de este procedimiento y se revisan aspectos sobresalientes de la técnica.

\section{ABSTRACT}

Severe gastrointestinal bleeding originated in the jejunum and ileum is rare, but it constitutes a serious threat to life. In these circumstances, the fast and accurate location of the bleeding site is critical to prevent and/or reduce morbidity and mortality from hypovolemic shock. Endoscopic examination of the small intestine, even in elective conditions, requires sophisticated resources hard to get in most hospitals in our country, especially in the case of urgent situations. For these cases, an intraoperative enteroscopy practiced with gastroduodenoscope (a device currently available in many public and private health institutions) can be useful. This report describes a technique of intraoperative enteroscopy, with a brief report of three successful clinical cases diagnosed through this procedure; highlights of the technique are reviewed.

\section{INTRODUCCIÓN}

A fortunadamente, son poco frecuentes ( $5 \%$ de los casos) los pacientes que presentan hemorragias digestivas graves (pérdida de 30 a $40 \%$ de la volemia) con gastroduodenoscopia y colonoscopia normales, pero constituyen un serio problema diagnóstico y terapéutico, con un riesgo importante para la vida por choque hipovolémico en caso de no detectarse rápida- mente el sitio del sangrado y lograr su control definitivo. Esto es particularmente cierto en áreas geográficas donde no existe disponibilidad inmediata de arteriografía, gammagrafía, angiotomografía computarizada o cápsula endoscópica. Aun cuando se cuenta con estos avanzados recursos tecnológicos, los mismos son de mayor utilidad en individuos hemodinámicamente estables. En circunstancias con un alto grado de urgencia por la magnitud del 
sangrado y carencia de recursos diagnósticos sofisticados, la enteroscopia transoperatoria es un excelente recurso diagnóstico (rendimiento del 70 al $100 \%$ ), con la ventaja adicional de que durante la laparotomía, una vez localizado el sitio y la causa del sangrado, es posible realizar el procedimiento quirúrgico adecuado para el control definitivo de la hemorragia. Se describe una técnica de enteroscopia transoperatoria empleando un gastroduodenoscopio y se presentan tres casos de hemorragia grave con origen en el intestino delgado diagnosticados a través de este procedimiento en el Hospital Privado Médica Farallón de Acapulco, Guerrero. $^{1-3}$

\section{DESCRIPCIÓN DE LA TÉCNICA}

Se efectúa la reanimación del paciente con cristaloides, coloides y sangre, según sea necesario, hasta lograr su máxima estabilidad. A continuación, se realiza una incisión media supra-peri-infraumbilical de $10 \mathrm{~cm}$ de longitud, se aborda la cavidad abdominal y se exterioriza el intestino delgado. En seguida, se lleva a cabo una enterotomía longitudinal de $1.5 \mathrm{~cm}$ en el borde antimesentérico del yeyuno, a $30 \mathrm{~cm}$ del ángulo de Treitz, y se introduce en forma retrógrada un gastroduodenofibroscopio previamente esterilizado en glutaraldehído, a fin de revisar la tercera y cuarta porción del duodeno, así como el yeyuno proximal. Posteriormente, por la misma enterotomía, se revisa el yeyuno distal introduciendo el fibroscopio en toda su longitud. La segunda enterotomía se realiza en el punto distal del yeyuno o íleon que fue alcanzado durante la primera enteroscopia. Usualmente son suficientes dos enterotomías para revisar todo el intestino delgado, pero si no fuese así, podría hacerse una tercera para alcanzar la visualización hasta la válvula ileocecal. La enteroscopia puede ser llevada a cabo por el mismo cirujano, si tiene el entrenamiento respectivo. Durante el procedimiento, el primer ayudante manipula con gentileza el intestino para facilitar el avance del fibroscopio alineando las asas intestinales; el segundo ayudante debe controlar manualmente el sitio de la enterotomía en tanto se avanza el fibroscopio para evitar o limitar fugas de líquido intestinal y disminuir la contaminación de la cavidad peritoneal, así como evitar desgarros de la enterotomía durante la enteroscopia. Una vez identificada la causa y sitio de la hemorragia, éste se refiere con un punto seromuscular de seda 3-0 y se determina el procedimiento quirúrgico apropiado para su control. Antes de cerrar la pared abdominal, debe lavarse la cavidad abdominal, por la contaminación que se haya generado durante la enteroscopia. Para evitar infecciones de la pared abdominal, es necesario cubrir con compresas húmedas los bordes de la herida quirúrgica y lavar por planos con una solución antiséptica al cerrar la herida.

\section{CASOS CLÍNICOS}

Caso 1. Paciente femenino de 42 años de edad, que ingresó en estado de choque hipovolémi$\mathrm{co}$, con hemoglobina de $3 \mathrm{~g} / \mathrm{dl}$, por hemorragia digestiva de una semana de evolución, con gastroduodenoscopia y colonoscopia previas normales. Se estabilizó hemodinámicamente y se efectuó laparotomía exploradora urgente, realizando enteroscopia transoperatoria con fibrogastroduodenoscopio; se localizó el sitio de sangrado en el yeyuno a $40 \mathrm{~cm}$ del ángulo de Treitz, proveniente de una angiodisplasia, se refirió el sitio del sangrado con un punto de seda 3-0, se realizó enterotomía longitudinal de $3 \mathrm{~cm}$ a este nivel y se controló la hemorragia mediante puntos de sutura por transfixión con seda 3-0. Las enterotomías se cerraron transversalmente en dos planos con puntos separados de seda 3-0. Se lavó la cavidad abdominal y se cerró la pared abdominal por planos. No hubo recurrencia del sangrado. No obstante, la mujer falleció dos semanas después por complicaciones derivadas del estado de choque grave y prolongado que la condujeron a falla orgánica múltiple.

Caso 2. Individuo masculino de 76 años de edad, con antecedente de ingestión reciente de AINE por osteoartritis, que ingresó por hemorragia digestiva grave de tres días de evolución; hemoglobina de $7 \mathrm{~g} / \mathrm{dl}$. Se le efectuaron gastroduodenoscopia y colonoscopia, que resultaron normales. Se estabilizó con cristaloides, coloides y sangre. En seguida se realizó laparotomía exploradora con enteroscopia transoperatoria, y se localizó el sitio de sangrado en un segmento del íleon de $15 \mathrm{~cm}$ de 
longitud que presentaba múltiples ulceraciones con hemorragia activa, ubicado a $20 \mathrm{~cm}$ de la válvula ileocecal. Se efectuó resección de este segmento (Figura 1) y anastomosis términoterminal. Las enterotomías de la enteroscopia se cerraron transversalmente en dos planos con puntos separados de seda 3-0. Se lavó la cavidad abdominal y se cerró la pared abdominal por planos. El reporte histopatológico fue de ileítis aguda con erosión superficial. La evolución postoperatoria transcurrió sin complicaciones. A nueve meses de su cirugía, no ha habido recurrencia del sangrado.

Caso 3. Paciente femenino de 84 años de edad, con antecedente de ingestión crónica de AINE por osteoartritis, que ingresó por hemorragia digestiva grave de una semana de evolución; hemoglobina de $5 \mathrm{~g} / \mathrm{dl}$, con gastroduodenoscopia y colonoscopia previas normales. Se estabilizó con cristaloides, coloides y sangre. En seguida se realizó laparotomía

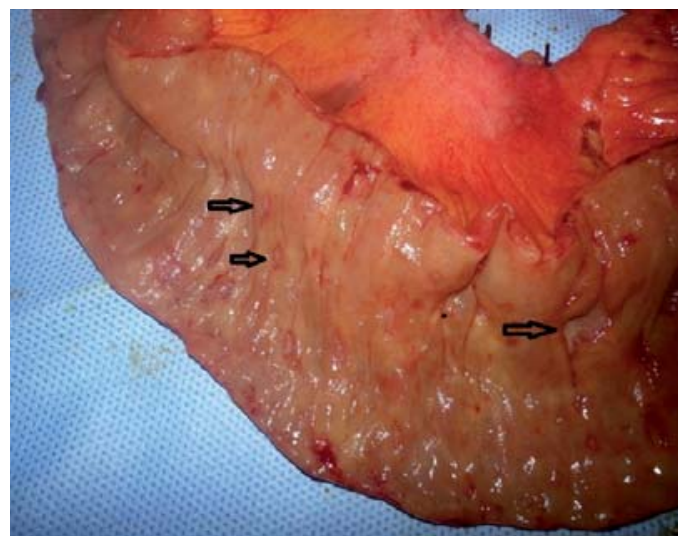

Figura 1. Segmento de íleon con múltiples ulceraciones.

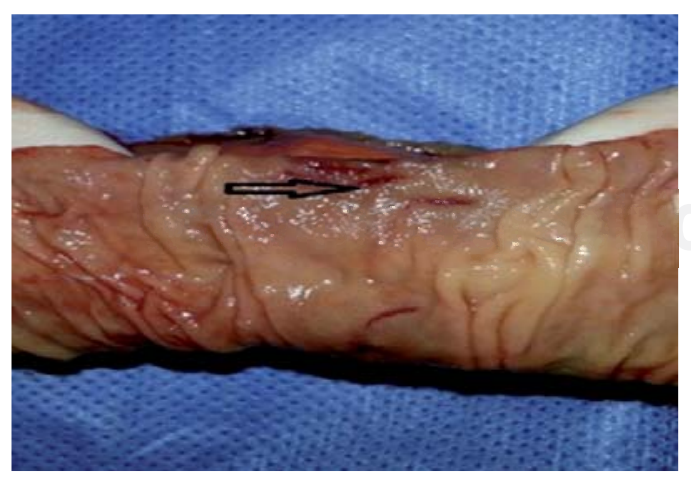

Figura 2. Segmento de íleon con múltiples ulceraciones. exploradora con enteroscopia transoperatoria, se localizó el sitio de sangrado en los últimos $15 \mathrm{~cm}$ del íleon terminal, segmento que presentaba múltiples ulceraciones sangrantes con hemorragia activa. Se efectuó resección de este segmento (Figura 2), ileostomía y cierre distal en bolsa de Hartmann. Las enterotomías de la enteroscopia se cerraron transversalmente en dos planos con puntos separados de seda 3-0. Se lavó la cavidad abdominal y se cerró la pared abdominal por planos. El reporte histopatológico fue de ileítis aguda con fisuras mucosas diseminadas. Tres meses después, se reconectó el intestino mediante anastomosis íleo-transversa. A ocho meses de su cirugía, no ha presentado recurrencia del sangrado.

\section{DISCUSIÓN}

En nuestro país es muy limitada la disponibilidad inmediata de recursos tecnológicos sofisticados para el diagnóstico y tratamiento de la hemorragia digestiva grave originada en el yeyuno e íleon, tales como la arteriografía, la gammagrafía, la angiotomografía computarizada o la cápsula endoscópica. Estos recursos sólo se encuentran en los grandes centros hospitalarios públicos y privados de algunas de las más grandes ciudades, y no siempre (turnos vespertinos, nocturnos, fines de semana, días festivos, vacaciones de especialistas, equipos en mantenimiento, etcétera).

Por otra parte, sí es factible disponer con relativa facilidad de gastroduodenofibroscopios en la mayor parte de las poblaciones que cuentan con hospitales de segundo nivel de atención médica.

Enfrentarse a una hemorragia grave del aparato digestivo de origen oculto constituye un verdadero reto para cualquier cirujano, ya que pone a prueba su ingenio y capacidad para la toma de decisiones en forma por demás rápida, considerando la infraestructura humana, de equipo y material con que cuenta en su sitio de trabajo para resolver en forma definitiva la causa del sangrado y salvar la vida del sujeto. ${ }^{4-6}$

Empleando la técnica de enteroscopia transoperatoria descrita en este reporte fue posible diagnosticar con exactitud el sitio de sangrado en los casos presentados y, consecuentemente, controlar la hemorragia con un 
procedimiento quirúrgico definitivo, ya que no se presentaron recurrencias. Si bien la persona del caso 1 falleció, su muerte fue consecutiva a complicaciones derivadas del estado de choque grave y prolongado que presentaba desde antes de su ingreso. Ninguno de los tres casos desarrolló complicaciones intraabdominales (íleo, sepsis, etcétera) ni infecciones de la pared abdominal. Por lo anterior, es razonable considerar que en la hemorragia digestiva grave originada en el intestino delgado, con un paciente adecuadamente resucitado, la enteroscopia transoperatoria empleando un gastroduodenofibroscopio previamente esterilizado en glutaraldehído es un procedimiento confiable para establecer el diagnóstico preciso de sitio y causa del sangrado; además, si la enteroscopia se realiza con alto grado de refinamiento técnico, cuidando los detalles señalados en este reporte, el procedimiento es muy seguro y no tiene por qué generar complicaciones. En otros reportes empleando colonoscopio se han señalado diversas morbilidades (hematoma de la pared intestinal, hemorragia mesentérica, íleo prolongado, isquemia intestinal y perforación); $;{ }^{7,8}$ esto probablemente se debe al mayor calibre y longitud del colonoscopio, lo que dificulta su manipulación y predispone a las complicaciones mencionadas.

La enteroscopia transoperatoria consume, en promedio, 45 minutos de tiempo quirúrgico, incluyendo el cierre de las enterotomías con puntos de sutura; en caso de contar con engrapadoras, este tiempo puede reducirse en aproximadamente 15 minutos.

En esta serie, la enteroscopia fue realizada por el mismo cirujano. Dadas las condiciones socioeconómicas de nuestro país, en donde la escasez de recursos humanos y tecnológicos de las áreas de la salud es la situación más común cotidianamente, resulta conveniente que los cirujanos generales cuenten con adiestramiento para la realización de gastroduodenoscopias; de esta forma, estarán en posibilidad de incrementar sus capacidades diagnósticas y terapéuticas en beneficio de la población de su área de influencia, particularmente en la resolución de urgencias quirúrgicas que amenazan la vida de las personas, como es el caso de las hemorragias graves del aparato digestivo originadas en el yeyuno e íleon.
Es de hacer notar que en los casos 2 y 3, los pacientes eran de edad avanzada y su único antecedente importante era la ingestión previa de AINE; en ambos, el sitio de hemorragia se desarrolló en un segmento del íleon terminal que presentaba múltiples ulceraciones. En los dos casos, el sangrado se controló con la resección del segmento intestinal afectado; en el caso 2 fue posible restablecer la continuidad intestinal en el mismo acto quirúrgico, ya que los últimos $20 \mathrm{~cm}$ del íleon estaban libres de patología. En el caso 3 fue necesario efectuar ileostomía y cierre distal en bolsa de Hartmann, en razón de que las lesiones ulcerativas comprendían hasta $2 \mathrm{~cm}$ antes de la válvula ileocecal, lo que hacía riesgoso realizar una anastomosis. Al respecto, en otras comunicaciones se ha informado que en individuos mayores de 40 años, la causa más frecuente de sangrado de intestino delgado son las lesiones vasculares tipo angiectasias, seguida por las enteropatías consecutivas al uso de AINE.6,7

\section{CONCLUSIÓN}

En hospitales que no cuentan con tecnología de vanguardia para el diagnóstico de la hemorragia digestiva aguda grave de intestino medio, pero que sí cuentan con un gastroduodenofibroscopio, como ocurre en la mayoría de los hospitales de segundo nivel de este país, la enteroscopia transoperatoria es un excelente recurso diagnóstico, con el valor agregado de que durante la laparotomía, una vez identificados la causa y el sitio de la hemorragia, se determina y realiza de inmediato el procedimiento quirúrgico apropiado para su control. Empleando una técnica depurada como la descrita en este reporte, el procedimiento no debe generar complicaciones ni prolongar en exceso el tiempo quirúrgico.

\section{REFERENCIAS}

1. Jakobs R, Hartmann D, Benz C, Schilling D, Weickert $\mathrm{U}$, Eickhert A, et al. Diagnosis of obscure gastrointestinal bleeding by intra-operative enteroscopy in 81 consecutive patients. World J Gastroenterol. 2006; 12: 313-316.

2. Pennazio M. Enteroscopy in the diagnosis and management of obscure gastrointestinal bleeding. Gastrointest Endosc Clin N Am. 2009; 19: 409-426. 
3. Lauren G, Ahmad K. Cost-effectiveness analysis of management strategies for obscure Gl bleeding. Gastrointest Endosc. 2008; 68: 920-936.

4. Baños JF. Sangrado gastrointestinal oscuro: ¿Cuál es el mejor abordaje diagnóstico? Rev Col Gastroenterol. 2010; 25: 177-184.

5. Pérez RF, González CP, Villafañez GMC, Picazo YJ, Lucendo VA. Enteroscopia total intraoperatoria urgente con colonoscopio en hemorragia digestiva baja grave mediante doble enterotomía. Cir Esp. 2009; 86: 252 253.

6. Monsanto P, Almeida N, Lérias C, Figuereido P, Gouveia $\mathrm{H}$, Sofia C. Is there a role for intraoperative enteroscopy in patients with obscure gastrointestinal bleeding? Rev Esp Enferm Dig. 2012; 104: 190-196.
7. Gómez RBJ, Romero CR, Hergueta DP, Pellicer BFJ, Herrerías GJM. Rendimiento de la enteroscopia. Rev Med Hosp Gen Mex. 2002; 65: 201-206.

8. Kopácová M, Bures J, Vykouril L, Hladik P, Simkovic D, Jon B, et al. Intraoperative enteroscopy: ten years' experience at a single tertiary center. Surg Endosc. 2007; 21: 1111-1116.

Correspondencia:

\section{Mauro Soto Granados}

Av. las Conchas 16-B,

Fraccionamiento Farallón, 39690, Acapulco, Guerrero, México.

Tel. (744) 4878790, Cel. 7444020225.

E-mail: m.d.maurosoto@hotmail.com 